\title{
Sulfatos en el cemento portland y su incidencia sobre el falso fraguado: Estado actual del conocimiento
}

IGNACIO DE LA CRUZ $\left({ }^{+}\right)$- TOMAS VAZQUEZ - OLGA FERNANDEZ-PEÑA

IETCC/CSIC

\section{$R E S U M E N$}

Se realiza un estudio bibliográfico de los sulfatos que pueden estar presentes en el clínker y cemento portland, asi como de los efectos de la aireación y temperatura sobre el fraguado.

Este trabajo es la fase previa de una amplia investigación experimental realizada

en el IETCC, sobre anomalias de fraguado

y fenómenos de "aterronamiento" en el cemento portland.

\section{$S U M M A R Y$}

A bibliographical study is carried out of the sulphates which may be present in the clinker and Portland cement, as likewise the effects of the aeration and temperature on the setting.

This work is a prior phase of a wide experimental investigation carried out in the IETCC, on anomalies or setting and phenomena of "lumping" in Portland cement.

\section{A N T ECEDENTES}

En el Instituto Eduardo Torroja se está realizando un trabajo de investigación en el cual, a partir de clínkeres industriales, se estudian fenómenos de falso fraguado y de "aterronamiento" de cementos.

La investigación está parcialmente concluida y dará lugar a posteriores publicaciones.

Forma parte de este estudio el falso fraguado que se observa en ocasiones en cementos aireados y la determinación de los sulfatos a través de espectroscopía IR $(*)$.

Con respecto a los sulfatos que pueden estar presentes en el clinker y cemento portland, el estudio se realizó a partir de mezclas binarias, ternarias y cuaternarias de los compuestos. Los análisis se hicieron cuali, semicuanti y cuantitativos (en algunos casos). Se determinó, asimismo, la influencia de la proporción y clase de esos sulfatos sobre la aparición del falso fraguado y la incidencia de la aireación del cemento sobre el fenómeno.

Finalmente se explicó el mecanismo causante del falso fraguado en cementos aireados y se adelantaron algunas soluciones que podrian impedir la aparición del fenómeno.

El estudio bibliográfico asi como comentarios sobre las investigaciones más importantes de los

(') Direccion actual: "Fibrotubo-Fibrolit. S. A." Carretera General de Andalucia Km. 30,200. VALDEMORO (Madrid).

(*) Esta parte del trabajo ha dado lugar a la Tesis de Licenciatura en Ciencias Quimicas de Pablo Ignacio de la Cruz. El presente articulo forma parte del estudio bibliográfico de las investigaciones que se han considerado más interesantes de las realizadas hasta la fecha. 
efectuadas hasta hoy, forman un "cuerpo de doctrina" que se ha considerado oportuno publicar aislada y previamente a la labor experimental desarrollada.

\section{SULFATOS EN EL CLINKER}

\section{Formación}

Antes de que se identificasen los sulfatos alcalinos en clínkeres (1), se creía que el $\mathrm{SO}_{3}$ estaba combinado con parte del $\mathrm{CaO}$ como $\mathrm{CaSO}_{4}$, pero desde su identificación se ha estudiado ampliamente la relación entre el $\mathrm{SO}_{3}$ y los álcalis en el clínker.

En los últimos años se ha incrementado la importancia de la conducta del azufre durante la calcinación del clínker debido, tanto al aumento en el uso de materias primas con mayor contenido en azufre, como a la subida del coste del fuel que ha dado lugar al uso de fuentes de energía de peor calidad y más ricas en $\mathrm{S}$.

En las materias primas el azufre suele encontrarse como (2): sulfatos (yeso y anhidrita), sulfuros (principalmente $\mathrm{FeS}_{2}$ ) y compuestos orgánicos. En el fuel-oil y el carbón el contenido puede exceder del $4 \%$. La conducta posterior del azufre está condicionada a la forma en que se encuentre combinado, así, cuando está en forma de sulfuros se oxida a $\mathrm{SO}_{2}$, mientras que en forma de sulfatos se produce $\mathrm{SO}_{3}$ que se descompone:

$$
\mathrm{SO}_{3} \rightarrow \mathrm{SO}_{2}+\frac{1}{2} \mathrm{O}_{2}
$$

Debido a que en la atmósfera del horno también se encuentran los álcalis evaporados, que tienen una afinidad alta por el azufre, se forman sulfatos alcalinos más resistentes a la disociación que el $\mathrm{CaSO}_{4}$. Este último se forma cuando debido a la baja volatilidad de los compuestos alcalinos o a su bajo contenido no hay suficientes álcalis en la atmósfera gaseosa para combinarse quimicamente con todo el $\mathrm{SO}_{2}$.

Por lo tanto los sulfatos alcalinos y cálcico se forman mientras es tomado el oxígeno en las reacciones:

$$
\begin{aligned}
& \mathrm{Na}_{2} \mathrm{O}+\mathrm{SO}_{2}+\frac{1}{2} \mathrm{O}_{2} \longleftrightarrow \mathrm{Na}_{2} \mathrm{SO}_{4} \\
& \mathrm{~K}_{2} \mathrm{O}+\mathrm{SO}_{2}+\frac{1}{2} \mathrm{O}_{2} \longleftrightarrow \mathrm{K}_{2} \mathrm{SO}_{4} \\
& \mathrm{CaO}+\mathrm{SO}_{2}+\frac{1}{2} \mathrm{O}_{2} \longleftrightarrow \mathrm{CaSO}_{4} \\
& \mathrm{CaCO}_{3}+\mathrm{SO}_{2}+\frac{1}{2} \mathrm{O}_{2} \longleftrightarrow \mathrm{CaSO}_{4}+\mathrm{CO}_{2}
\end{aligned}
$$

Normalmente se ha considerado la presencia de estos sulfatos en forma de sales dobles. Así, NEWKIRK (3) dice que si ambos álcalis, $\mathrm{Na}$ y $\mathrm{K}$, están presentes junto con $\mathrm{SO}_{3}$, se forma un sulfato alcalino que contiene $\mathrm{K}_{2} \mathrm{O}$ y $\mathrm{Na}_{2} \mathrm{O}$, de manera que si el número de moles de $\mathrm{SO}_{3}$ disponible es menor que el número total de moles de álcalis, estos tienen tendencia a combinarse con todo el $\mathrm{SO}_{3}$ en la relación molar $\mathrm{K}_{2} \mathrm{O}: \mathrm{Na}_{2} \mathrm{O}=3$, dependiendo de la disponibilidad de los reactivos en la mezcla. El exceso de álcalis reacciona con otros componentes para dar $\mathrm{NC}_{8} \mathrm{~A}_{3}$ y $\mathrm{KC}_{23} \mathrm{~S}_{12}$. Si hay exceso de $\mathrm{SO}_{3}$ estará como $\mathrm{CaSO}_{4}$.

POLLIT Y BROWN (4) también indican la afinidad del azufre hacia los álcalis, preferentemente el potasio. Muestran la existencia de la disolución sólida continua en las mezclas calentadas de $\mathrm{Na}_{2} \mathrm{SO}_{4}$, por lo que piensan que en un clinker el $\mathrm{Na}_{2} \mathrm{SO}_{4}$ estará en forma de doble sulfato alcalino con $\mathrm{K}_{2} \mathrm{SO}_{4}$. $\mathrm{Si}$ hay $\mathrm{SO}_{3}$ en exceso puede estar presente ya como $\mathrm{CaSO}_{4}$ o como $2 \mathrm{CaSO}_{4} \cdot \mathrm{K}_{2} \mathrm{SO}_{4}$. 
Según MANDER y SKALNY (5) la formación de una u otra sal doble depende de las condiciones del horno, formándose preferentemente langbeinita cálcica $\left(2 \mathrm{CaSO}_{4} \cdot \mathrm{K}_{2} \mathrm{SO}_{4}\right)$ en condiciones oxidantes y aphtitalita $\left(3 \mathrm{~K}_{2} \mathrm{SO}_{4} \cdot \mathrm{Na}_{2} \mathrm{SO}_{4}\right)$ en condiciones reductoras.

GUTT Y SMITH (6) en un estudio de los sulfatos en el clínker de cemento portland comprobaron que en mezclas en las que había exceso de iones sulfato con respecto a los álcalis, el $\mathrm{K}_{2} \mathrm{O}$ reaccionaba en todos los casos con el $\mathrm{CaSO}_{4}$ formando langbeinita. También estudiaron el comportamiento del $\mathrm{CaSO}_{4}$ en la manufactura del clinker portland en ausencia de álcalis y magnesia, dándose en este caso la formación de sulfatos complejos: sulfo-spurrita $\left(2 \mathrm{C}_{2} \mathrm{~S}_{\text {.CaSO }}\right)$ y sulfoaluminato $\left(3 \mathrm{CA} \cdot \mathrm{CaSO}_{4}\right)$. estas sales complejas, junto a la langbeinita y otras sales dobles formadas por reacciones con los gases de horno $\left(\mathrm{SO}_{2}\right.$ y $\left.\mathrm{CO}_{2}\right)$ dan lugar a la formación de costras, anillos y pegaduras (7) $(*)$.

\section{Determinaciones potenciales}

A partir del análisis de $\mathrm{K}_{2} \mathrm{O}, \mathrm{Na}_{2} \mathrm{O}$ y $\mathrm{SO}_{3}$ del clinker OSBAECK (8) hace una estimación potencial de los sulfatos alcalinos. NEWKIRK (3) desarrolló las ecuaciones para indicar el efecto de los álcalis en la composición potencial del clínker de manera que por el análisis químico se puede dar la composición de éste en los sulfatos (alcalinos y cálcico) y del resto de los minerales del clínker.

\section{SULFATOS EN CEMENTOS}

\section{Reguladores}

Para evitar la rápida hidratación del $\mathrm{C}_{3} \mathrm{~A}$ del clínker de forma que el fraguado sea normal, se añaden reguladores del fraguado, que forman compuestos insolubles con el $\mathrm{C}_{3} \mathrm{~A}$ recubriendo a éste e impidiendo su hidratación. Los reguladores más empleados suelen ser los sulfatos cálcicos en cualquiera de sus formas. La adición de éstos data de los principios de la fabricación del cemento portland y es atribuida por unos a Scott y por otros a Michaelis.

Ya en 1926 MEADE (9) probó que tanto el yeso $\left(\mathrm{CaSO}_{4} \cdot 2 \mathrm{H}_{2} \mathrm{O}\right)$, como el hemihidrato $\left(\mathrm{CaSO}_{4} \cdot \frac{1}{2} \mathrm{H}_{2} \mathrm{O}\right)$, como la anhidrita natural $\left(\beta-\mathrm{CaSO}_{4}\right)$ regulan el fraguado, aunque la proporción necesaria para hacerlo varía de uno a otro.

MARGARIT (10) afirma que entre las diversas formas de sulfato cálcico, cuatro son las que pueden ser utilizadas como reguladores: yeso, hemihidrato, anhidrita artificial o soluble $\left(\gamma-\mathrm{CaSO}_{4}\right)$ y la anhidrita natural, y concluye:

1. De todos, el yeso es el regulador más seguro y eficaz. Como a temperaturas relativamente

$\left({ }^{*}\right)$ Alcalis.

El papel que puede jugar el $\mathrm{Na}^{+}$y el $\mathrm{K}^{+}$en el fraguado y en la hidratación del cemento es muy distinto. Ya se ha mencionado la formación de los diversos sulfatos alcalinos, en forma de sales sencillas o dobles, durante la clinkerización y la hidratacióil. Asi como el $\mathrm{K}^{+}$tiene preferencia para combinarse formando $\mathrm{SO}_{4} \mathrm{~K}_{2}$ o bien $2 \mathrm{SO}_{4} \mathrm{Ca}$. SO $\mathrm{SO}_{2}$, el $\mathrm{Na}^{+}$preferentemente se incorpora en la red del $\mathrm{C}_{3} \mathrm{~A}$, formando disoluciones sólidas que hacen variar la estructura cristalina del aluminato, desde cúbico (I) $\left(\mathrm{C}_{3} \mathrm{~A}\right.$ puro) hasta monoclinico o incluso $\mathrm{NC}_{8} \mathrm{~A}_{3}$. La proporción en la cual el $\mathrm{Na}^{+}$ puede entrar en la red de los aluminatos varia en caso de que además ese aluminato lleve incorporado otros iones extraños, principalmente $\mathrm{Si}^{4 \dagger}$.

Asimismo es de resaltar que, según SKIN Y GLASSER (54), el reparto de los álcalis $\mathrm{Na}$ y $\mathrm{K}$ en las fases del clinker es función tanto de la cantidad de álcalis, como de la proporción en que se encuentran uno con respecto a otro. Por ello se puede deducir que en el caso de una baja relación $\mathrm{Na} / \mathrm{K}$, el clinker tendrá aluminato cúbico y probablemente se forme una fase del tipo $\mathrm{KC}_{23} \mathrm{~S}_{12}$. 
bajas pierde parte del agua de cristalización es recomendable mantener, durante el proceso de molienda del clínker con el yeso, la temperatura de los molinos por debajo de $70^{\circ} \mathrm{C}$.

2. Con el hemihidrato y la anhidrita soluble también se puede regular el fraguado. En estos casos la adición de regulador no debe rebasar cierto límite. Con un $3 \%$ existe ya el peligro de que el cemento adquiera falso fraguado.

3. La anhidrita natural viene a ser el menos adecuado de todos los tipos de sulfato cálcico para regular el fraguado.

Tećnicamente la actuación de cada una de las variedades depende de su solubilidad de manera que las más solubles, el hemihidrato y la anhidrita soluble (11) regulan con mayor eficacia que el yeso y la anhidrita insoluble. Sin embargo, si se incrementan las cantidades de hemihidrato y anhidrita soluble se puede dar el fenómeno del falso fraguado por precipitación de cristales de yeso.

\section{FORMADOS POSTERIORMENTE}

El regulador añadido normalmente al clínker es el yeso, pero éste según las condiciones del molido puede deshidratarse a $\beta-\mathrm{CaSO}_{4} \cdot \frac{1}{2} \mathrm{H}_{2} \mathrm{O}$ o incluso a $\gamma-\mathrm{CaSO}_{4}$, dependiendo esta transformación tanto del tiempo como de la temperatura del molido y de la humedad relativa (12). Hay numerosos estudios de los productos de deshidratación del yeso con la temperatura, utilizando las técnicas de ATD, difracción de rayos X y espectroscopía infrarroja (13-18).

Además de esta deshidratación hay otras transformaciones que se pueden dar en los sulfatos presentes en el cemento debidas al agua utilizada en el enfriamiento del molino, o posteriormente, a las condiciones de almacenamiento en las que se pueden dar tanto deshidrataciones (por temperaturas elevadas y baja humedad relativa) como ligeras hidrataciones por aireación.

Un sulfato que aparece por estas causas es la singenita $\left(\mathrm{CaSO}_{4} \cdot \mathrm{K}_{2} \mathrm{SO}_{4} \cdot \mathrm{H}_{2} \mathrm{O}\right)$, a la que se suele atribuir la formación de grumos en el cemento. Su presencia se puede explicar:

- Por la transformación de la langbeinita que lleva el clínker, debido a la humedad atmosférica (19):

$$
2 \mathrm{CaSO}_{4} \cdot \mathrm{K}_{2} \mathrm{SO}_{4}+\mathrm{H}_{2} \mathrm{O} \rightarrow \mathrm{K}_{2} \mathrm{SO}_{4} \cdot \mathrm{CaSO}_{4} \cdot \mathrm{H}_{2} \mathrm{O}+{ }^{\prime C} \mathrm{CaSO}_{4} \text { " }
$$

Este "CaSO 4 " activo pasa de forma inmediata a $\mathrm{CaSO}_{4} \cdot \frac{1}{2} \mathrm{H}_{2} \mathrm{O}$, según:

$$
\mathrm{CaSO}_{4}+\frac{1}{2} \mathrm{H}_{2} \mathrm{O} \rightarrow \mathrm{CaSO}_{4} \cdot \frac{1}{2} \mathrm{H}_{2} \mathrm{O}
$$

- En el molido del cemento si éste tiene un elevado contenido en $\mathrm{K}_{2} \mathrm{SO}_{4}$ y a temperaturas de $80-160^{\circ} \mathrm{C}$ en presencia de vapor de agua originado por la inyección de agua en el molino (20). Se forma preferentemente en cementos que contienen sulfato cálcico hemihidratado:

$$
\mathrm{CaSO}_{4} \cdot \frac{1}{2} \mathrm{H}_{2} \mathrm{O}+\mathrm{K}_{2} \mathrm{SO}_{4}+\frac{1}{2} \mathrm{H}_{2} \mathrm{O} \rightarrow \mathrm{CaSO}_{4} \cdot \mathrm{K}_{2} \mathrm{SO}_{4} \cdot \mathrm{H}_{2} \mathrm{O}
$$

- Por las condiciones de almacenamiento, ya que si éste se hace a temperaturas elevadas puede proseguir la deshidratación del yeso, y el agua desprendida da lugar a la formación de singenita (21) (22). También en almacenamientos en los que la humedad relativa sea elevada (23) (24) (25).

Otro tipo de transformación en los sulfatos, que se produce si las condiciones son de humedad 
relativa muy elevada o en el caso de aireaciones muy prolongadas, es la formación de ettringita a partir de los sulfatos cálcicos y los aluminatos (22) (25) (26):

$$
\mathrm{C}_{3} \mathrm{~A}+\mathrm{CaSO}_{4} \cdot 2 \mathrm{H}_{2} \mathrm{O}+30 \mathrm{H}_{2} \mathrm{O} \rightarrow \mathrm{C}_{3} \mathrm{~A} .3 \mathrm{CaSO}_{4} .32 \mathrm{H}_{2} \mathrm{O}
$$

También se podrá dar a partir del $\mathrm{CaSO}_{4} \cdot \frac{1}{2} \mathrm{H}_{2} \mathrm{O}$.

\section{Detección y análisis}

Fue Brown en 1936 el primero en detectar, en polvos tomados del recubrimiento del horno, la presencia de una fase identificada como sulfato alcalino. TAYLOR (1) en 1942 identificó como $\mathrm{K}_{2} \mathrm{SO}_{4}$ una fase encontrada en clinkeres comerciales; hasta entonces se había supuesto que el $\mathrm{SO}_{3}$ de los clínkeres estaba combinado con parte del $\mathrm{CaO}$ como $\mathrm{CaSO}_{4}$.

En la actualidad son varias las técnicas que se emplean para el estudio de los sulfatos en clinkeres y cementos: DRX, IR, ATD, ATG y una combinación de SEM y EDS (microscopia electrónica y espectroscopia de energía dispersiva).

El ATD se utiliza para el análisis de los sulfatos hidratados $\left(\mathrm{CaSO}_{4} \cdot 2 \mathrm{H}_{2} \mathrm{O}, \mathrm{CaSO}_{4} \cdot \frac{1}{2} \mathrm{H}_{2} \mathrm{O}\right.$, $\mathrm{CaSO}_{4} \cdot \mathrm{K}_{2} \mathrm{SO}_{4} \cdot \mathrm{H}_{2} \mathrm{O}$ y ettringita), siendo de especial interés para el caso de hidrataciones y meteorizaciones, incluso para análisis cuantitativos (ATG) (21) (23) (25) (27) (28).

Por DRX también se analizan los sulfatos de clinkeres y cementos, aunque normalmente por la pequeña proporción de éstos, se ha de hacer en fracciones enriquecidas por disolución selectiva de los silicatos. ADAMS (29) y MANDER y colaboradores (30) hacen determinaciones cuantitativas de $\mathrm{K}_{2} \mathrm{SO}_{4}, 2 \mathrm{CaSO}_{4} \cdot \mathrm{K}_{2} \mathrm{SO}_{4}, \mathrm{CaSO}_{4}$ y $\mathrm{CaSO}_{4} \cdot 2 \mathrm{H}_{2} \mathrm{O}$ y semicuantitativas de singenita por este método. El estudio de estas fracciones enriquecidas por el método de Takashima de disolución selectiva de silicatos, ha llevado a la detección de otro sulfato doble que aparece en los clínkeres portland: la aphtitalita $\left(3 \mathrm{~K}_{2} \mathrm{SO}_{4} \cdot \mathrm{Na}_{2} \mathrm{SO}_{4}\right)$ o soluciones sólidas entre $\mathrm{K}_{2} \mathrm{SO}_{4}$ y $\mathrm{Na}_{2} \mathrm{SO}_{4}$, que pueden combinarse en cualquier proporción, detectada por YAMAGUCHI (31) al analizar el sulfato alcalino presente en la fase intersticial de un clínker y determinar, por medida del d-espaciado, su composición como $3 \mathrm{~K}_{2} \mathrm{SO}_{4} \cdot \mathrm{Na}_{2} \mathrm{SO}_{4}$ (ligeramente rico en K). MANDER y SKALNY (5) también encuentran aphtitalita en un clínker obtenido en condiciones reductoras. Asimismo se ha detectado en pastas de cemento hidratadas en las que se concentra en la superficie por "exudación o rezumado" (32).

La detección de la forma en que se encuentran los sulfatos en los clínkeres portland también se ha hecho por combinación de SEM y EDS, analizándose por esta técnica $2 \mathrm{CaSO}_{4} \cdot \mathrm{K}_{2} \mathrm{SO}_{4}$ y $\mathrm{K}_{2} \mathrm{SO}_{4}(5)(33)$.

Por último una técnica muy apropiada para el estudio de sulfatos en cementos y clinkeres es la espectroscopia IR. NIEL (34) en su tesis doctoral indica las bandas más adecuadas para el análisis de algunos sulfatos de cemento: $\mathrm{CaSO}_{4}, \mathrm{CaSO}_{4} \cdot \frac{1}{2} \mathrm{H}_{2} \mathrm{O}, \mathrm{CaSO}_{4} \cdot 2 \mathrm{H}_{2} \mathrm{O}, \mathrm{Na}_{2} \mathrm{SO}_{4} \cdot 10 \mathrm{H}_{2} \mathrm{O}$, $\mathrm{K}_{2} \mathrm{SO}_{4}$, singenita y ettringita. También proporciona un método para el análisis cuantitativo de mezclas de yeso, hemihidrato y anhidrita natural en cementos. VAZQUEZ estudia las diferencias entre los espectros de anhidrita natural, hemihidrato y yeso (36). En su tesis doctoral (35) estudia cementos conteniendo yeso, hemihidrato y singenita. BENSTED y VARMA (37) analizan los espectros IR de $\mathrm{K}_{2} \mathrm{SO}_{4}, \mathrm{Na}_{2} \mathrm{SO}_{4}$, langbeinita y singenita, así como de la ettringita, el monosulfoaluminato y de los diferentes sulfatos cálcicos. Estos últimos han sido objeto de numerosos estudios por espectroscopia IR (13) (15) (18) (38). 


\section{FRAGUADOS ANOMALOS}

El fraguado es el cambio de las caracteristicas de una pasta de cemento con aumento de la consistencia hasta adquirir las propiedades de un sólido.

El inicio y fin del fraguado se determina con la aguja de VICAT (39), aparato constituido por una varilla delgada, cilindrica, de metal pulimentado, terminada por una sección recta y lisa de $1 \mathrm{~mm}^{2}$ de superficie y cargada de modo que el peso total sea de 300 gramos.

El ensayo se hace con una pasta normal, que tiene una consistencia fijada según una determinada norma (39) colocada en un molde troncocónico normalizado de $40 \mathrm{~mm}$ de altura.

Se dice que ha empezado el fraguado cuando la penetración de la aguja en la pasta es de $35 \mathrm{~mm}$, y que ha terminado cuando penetra en la masa $5 \mathrm{~mm}$. Los tiempos transcurridos hasta el principio y fin del fraguado se cuentan a partir del instante en que empieza el amasado de la pasta. En los cementos portland para que se considere normal, el principio del fraguado ha de darse después de 45 minutos y el final antes de 12 horas.

Hay dos tipos de fraguados anómalos que vienen dados por un endurecimiento rápido y que se denominan "rápido" y "falso".

En el fraguado rápido la pasta endurece rápida e irreversiblemente con desprendimiento de calor al producirse la combinación con el agua. El falso fraguado también viene dado por un rápido aumento de la consistencia, aunque sin desprendimiento de calor; si se vuelve a amasar la pasta endurecida en este caso, se consigue que la consistencia de la pasta sea similar a la inicial.

\section{Causas del fraguado rápido}

El fraguado rápido se da en cementos que no contienen yeso u otro regulador del fraguado, de manera que al mezclar con agua se produce la hidratación de los aluminatos cálcicos, según las siguientes reacciones:

$$
\begin{aligned}
& 3 \mathrm{CaO} \cdot \mathrm{Al}_{2} \mathrm{O}_{3}+6 \mathrm{H}_{2} \mathrm{O} \rightarrow 3 \mathrm{CaO} \cdot \mathrm{Al}_{2} \mathrm{O}_{3} \cdot 6 \mathrm{H}_{2} \mathrm{O} \text { (Cúbico) } \\
& 3 \mathrm{CaO} \cdot \mathrm{Al}_{2} \mathrm{O}_{3}+13 \mathrm{H}_{2} \mathrm{O}+\mathrm{CaO} \rightarrow 4 \mathrm{CaO} \cdot \mathrm{Al}_{2} \mathrm{O}_{3} \cdot 13 \mathrm{H}_{2} \mathrm{O} \text { (Hexagonal) }
\end{aligned}
$$

La formación de uno u otro aluminato dependerá de la riqueza en cal de la fase liquida de la pasta de cemento. En cementos ricos en cal, es posible que sólo se forme el segundo. Estas reacciones desprenden el calor que se observa, y los hidratos producidos en ellas con los que confieren la rigidez a la pasta.

Un caso especial de fraguado rápido en cementos portland con elevado contenido en sulfato alcalino ha sido atribuido a la precipitación de singenita (40).

\section{Causas del falso fraguado}

Hay numerosas hipótesis para explicar este fenómeno /se han llegado a enumerar hasta treinta y ocho (41)|. La mayoria de ellas apuntan a la presencia en el cemento de sulfato cálcico hemihidrato o de anhidrita soluble, o de ambos, que se disuelven rápidamente en los primeros minutos del amasado precipitando como yeso.

Las razones por las que aparece el fenómeno del falso fraguado en los cementos se resumen en: 
- Temperaturas elevadas alcanzadas en la molienda conjunta del clínker y yeso con la consiguiente deshidratación de éste.

- Exposición al aire en ciertas condiciones de humedad relativa (aireación).

\section{Efecto de la temperatura}

Para determinados valores en las temperaturas alcanzadas se produce la deshidratación del yeso añadido al clínker como regulador, transformándose en hemihidrato y anhidrita soluble. Estos causan disoluciones sobresaturadas con respecto al yeso.

Según TURRIZIANI (42) en estas condiciones hay una cristalización rápida con el $\mathrm{C}_{3} \mathrm{~A}$, de ettringita, produciéndose una especie de fieltro causante del falso fraguado.

ENTINE (43) afirma también que el falso fraguado está relacionado con la estructura yeso-ettringita, estando a su vez condicionado por la fuerza iónica de la disolución dependiente de los sulfatos alcalinos. Estos influyen asimismo en la nucleación.

Para FRIGIONE (44) la precipitación y tamaño de cristales de yeso es la causante del atiesamiento. La forma en que se presenta éste depende de las temperaturas alcanzadas en la molienda: entre 90 y $130^{\circ} \mathrm{C}$ el desarrollo del falso fraguado es lento y desaparece con tiempos de mezcla de 2 a 3 minutos, mientras que para temperaturas entre 140 y $180^{\circ} \mathrm{C}$ es rápido y son necesarios de 6 a 8 minutos de amasado para su destrucción.

\section{Efecto de la aireación}

La aireación a la que puede estar sometido un cemento afecta en gran manera a las características de éste, influyendo tanto en las resistencias que pueden alcanzar a determinadas edades como a los tiempos de fraguado o incluso a la constitución química (formación de carbonatos, hidratos, etc.) (45), dando lugar también a la aparición o desaparición de fraguados anómalos de manera que se pueden dar los siguientes casos (46):

- Cementos que no presentan nunca falso fraguado, aún después de una larga exposición al aire.

- Cementos que presentan falso fraguado desapareciendo éste después de la aireación.

- Cementos que muestran falso fraguado después de aireados y en los que desaparecen por aireación prolongada.

Inicialmente la aparición del fenómeno del falso fraguado por aireación se atribuia a la carbonatación de los álcalis presentes en el cemento (47), siendo la causa la precipitación rápida de cristales de carbonato cálcico. Aunque se ha demostrado que la adición de carbonatos alcalinos o la presencia de éstos en el cemento pueden producir fraguados anómalos (48), retrasando la formación de ettringita y por precipitación de $\mathrm{CaCO}_{3}$, este caso no es el que se produce por aireación puesto que los sulfatos alcalinos del cemento no se transforman en carbonatos. Además ésto lo demostró experimentalmente GILLILAND (14) calentando un cemento aireado que tenía falso fraguado a una temperatura suficiente para transformar el $\beta-\mathrm{CaSO}_{4 \cdot \frac{1}{2}} \mathrm{H}_{2} \mathrm{O}$ en $\beta-\mathrm{CaSO}_{4}$, pero insuficiente para descomponer los hipotéticos carbonatos formados, desapareciendo de esta forma cl falso fraguado.

Posteriormente han surgido gran número de explicaciones para este fenómeno, atribuyéndose a causas muy diversas, aunque la aceptada generalmente es que este tipo de falso fraguado es también debido al yeso deshidratado. 
Para HANSEN (49) la aireación produce una inactivación del $\mathrm{C}_{3} \mathrm{~A}$ y $\mathrm{C}_{3} \mathrm{~S}$ dado que estos reaccionan con mucha facilidad con el agua y con el $\mathrm{CO}_{2}$, de manera que sus superficies queden recubiertas por los productos de reacción, impidiendo la rápida reacción del $\mathrm{C}_{3} \mathrm{~A}$ con el agua y el $\mathrm{CaSO}_{4}$ al mezclar con el agua. Por lo tanto el yeso deshidratado formado en la molienda producirá una sobresaturación de la fase liquida con cristalización de yeso. SELIGMAN y GREENING (50) afirman que la inactivación de los aluminatos frente a los sulfatos se

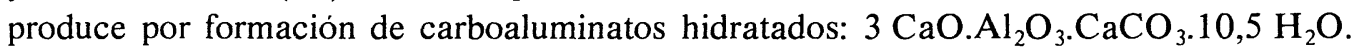

Según MANABE (51) la aireación reduce la acción aceleradora del clínker sobre la precipitación del yeso, aunque no varia el grado de combinación con los aluminatos. Al igual que RIO y TURRIZIANI (46) establecen que un cemento que de falso fraguado por aireación también lo da antes de ésta, aunque la precipitación del yeso ocurre durante el amasado, comprobándolo con tiempos de amasado menores. Estos mismos investigadores creen que la aireación varía las cantidades relativas de hemihidrato y yeso por transformación del primero en el segundo.

Según VAZQUEZ y FERNANDEZ-PEÑA (19), el falso fraguado por aireación se produce en cementos con hemihidrato y en los que se haya producido langbeinita cálcica durante la clínkerización, de forma que ésta reacciona con la humedad ambiente:

$$
\mathrm{K}_{2} \mathrm{SO}_{4} \cdot 2 \mathrm{CaSO}_{4}+\mathrm{H}_{2} \mathrm{O} \rightarrow \mathrm{K}_{2} \mathrm{SO}_{4} \cdot \mathrm{CaSO}_{4} \cdot \mathrm{H}_{2} \mathrm{O}+" \mathrm{CaSO}_{4} "
$$

El $\mathrm{CaSO}_{4}$ formado es muy activo y actúa de germen en la transformación del hemihidrato:

$$
\mathrm{CaSO}_{4} \cdot \frac{1}{2} \mathrm{H}_{2} \mathrm{O}+1,5 \mathrm{H}_{2} \mathrm{O} \stackrel{\mathrm{CaSO}_{4 \cdot \frac{1}{2}} \mathrm{H}_{2} \mathrm{O}}{\longrightarrow} \mathrm{CaSO}_{4} \cdot 2 \mathrm{H}_{2} \mathrm{O}
$$

Por lo tanto el falso fraguado aparecerá mientras se produzca la reacción I.

Para RICHARTZ (25), el almacenamiento de cementos con elevado contenido en aluminato alcalino produce la deshidratación del yeso a hemihidrato. Este recristaliza al mezclar con agua y junto con los cristales de ettringita y singenita que se forman simultáneamente se produce la solidificación. Posteriormente se descompone la estructura de cristales de yeso por la reacción con el $\mathrm{C}_{3} \mathrm{~A}$ y se puede recuperar la plasticidad anterior, a no ser que el contenido de ettringita o singenita sea muy grande.

Otras causas enumeradas por algunos investigadores y no relacionadas con las anteriores son:

- Floculación de los granos del cemento producida por la presencia de hidróxido cálcico formado durante la aireación (52)

- Por comportamiento tixotrópico de los granos de cemento parcialmente hidratados por la aireación (53) que causan el falso fraguado. En este caso el falso fraguado se dio en un cemento con yeso y $\sin \mathrm{C}_{3} \mathrm{~A}$.

\section{ANEJ O}

Como se ha visto en la bibliografía estudiada, los sulfatos que pueden aparecer en clinkeres y cementos portland anhidros, son los siguientes:

$-\mathrm{CaSO}_{4} \cdot 2 \mathrm{H}_{2} \mathrm{O} \ldots \ldots \ldots \ldots \ldots \ldots \ldots \ldots \ldots \ldots \ldots \ldots$ yeso

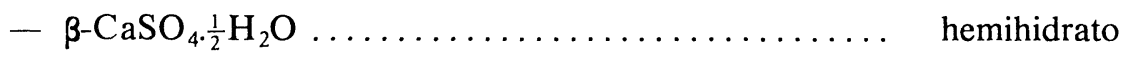

$-\beta-\mathrm{CaSO}_{4} \ldots \ldots \ldots \ldots \ldots \ldots \ldots \ldots \ldots \ldots \ldots \ldots \ldots \ldots$ anhidrita insoluble

$-\gamma-\mathrm{CaSO}_{4} \ldots \ldots \ldots \ldots \ldots \ldots \ldots \ldots \ldots \ldots \ldots \ldots \ldots \ldots$ anhidrita soluble 
$-\mathrm{K}_{2} \mathrm{SO}_{4}$

arcanita

$-\mathrm{Na}_{2} \mathrm{SO}_{4}$

thernardita

$-2 \mathrm{CaSO}_{4} \cdot \mathrm{K}_{2} \mathrm{SO}_{4}$

langbeinita cálcica

$-\mathrm{CaSO}_{4} \cdot \mathrm{K}_{2} \mathrm{SO}_{4} \cdot \mathrm{H}_{2} \mathrm{O}$

singenita

$-3 \mathrm{~K}_{2} \mathrm{SO}_{4} \cdot \mathrm{Na}_{2} \mathrm{SO}_{4}$

aphthitalita o disolución sólida similar

Los espectros IR, las frecuencias de absorción de las bandas correspondientes y sus asignaciones se incluyen en el presente trabajo. No obstante un estudio amplio de la espectroscopia IR de los compuestos citados se dará a conocer en una posterior publicación.

\begin{tabular}{|c|c|c|c|c|}
\hline $\mathbf{K}_{2} \mathbf{S O}_{\mathbf{4}}$ & $\mathbf{N a}_{2} \mathbf{S O}_{\mathbf{4}}$ & Apht. & Lang. & Asig. \\
\hline $1163 \mathrm{~h}$ & $1157 \mathrm{~h}$ & $* 1185 \mathrm{~F}$ & $* 1204 \mathrm{~d}$ & \\
$1144 \mathrm{~h}$ & $* 1129 \mathrm{~F}$ & $* 1110 \mathrm{~F}$ & $\nu_{3} \mathrm{SO}_{4}$ \\
$* 1115 \mathrm{~F}$ & $1102 \mathrm{~h}$ & & $\begin{array}{l}1149 \mathrm{~h} . \mathrm{a} \\
1125 \mathrm{~F} . \mathrm{a}\end{array}$ & \\
\hline $981 \mathrm{~d}$ & & & $1018 \mathrm{~d}$ & $\nu_{1} \mathrm{SO}_{4}$ \\
\hline$* 618 \mathrm{f}$ & $637 \mathrm{~m}$ & $617 \mathrm{f}$ & $643 \mathrm{~m}$ & \\
& $616 \mathrm{~m}$ & & $628 \mathrm{~m}$ & $\nu_{4} \mathrm{SO}_{4}$ \\
\hline
\end{tabular}

\begin{tabular}{|c|c|c|c|c|}
\hline Yeso & Hem. & Anh. Ins. & Singenita & Asig. \\
\hline $3550 \mathrm{f}$ & $* 3610 \mathrm{~m}$ & & \multirow{2}{*}{ * $3320 \mathrm{f}$} & $\nu_{3} \mathrm{H}_{2} \mathrm{O}$ \\
\hline $\begin{array}{l}* 3405 \mathrm{f} \\
3245 \mathrm{~d}\end{array}$ & $3550 \mathrm{~m}$ & & & $\nu_{1} \mathrm{H}_{2} \mathrm{O}$ \\
\hline $\begin{array}{l}* 1680 \mathrm{~m} \\
1620 \mathrm{~d}\end{array}$ & $1620 \mathrm{~m}$ & & 1680 d. a & $\nu_{2} \mathrm{H}_{2} \mathrm{O}$ \\
\hline $\begin{array}{l}1144 \mathrm{~F} \\
1120 \mathrm{~F}\end{array}$ & $\begin{array}{r}* 1153 \mathrm{~F} \\
1116 \mathrm{~m} \\
* 1094 \mathrm{f}\end{array}$ & $\begin{array}{l}* 1160 \mathrm{~F} \\
1122 \mathrm{~F}\end{array}$ & $\begin{array}{r}* 1191 \mathrm{~F} \\
1139 \mathrm{~F} \\
* 1125 \mathrm{~F} \\
1104 \mathrm{~F}\end{array}$ & $\nu_{3} \mathrm{SO}_{4}$ \\
\hline $1003 \mathrm{~d}$ & $1007 \mathrm{~d}$ & $1014 \mathrm{~d}$ & $\begin{array}{r}1000 \mathrm{~d} \\
979 \mathrm{~d}\end{array}$ & $\nu_{1} \mathrm{SO}_{4}$ \\
\hline & & & $751 \mathrm{~m}$ & flexion $\mathrm{M}-\mathrm{O}-\mathrm{H}$ \\
\hline $\begin{array}{l}\text { * } 669 \mathrm{~m} \\
601 \mathrm{~m}\end{array}$ & $\begin{array}{l}* 660 \mathrm{~m} \\
625 \mathrm{~h} \\
600 \mathrm{~m}\end{array}$ & $\begin{array}{l}* \quad 677 \mathrm{f} \\
613 \mathrm{~m} \\
* \quad 593 \mathrm{~m}\end{array}$ & $\begin{array}{l}\text { * } 657 \mathrm{~m} \\
643 \mathrm{~h} \\
602 \mathrm{r}\end{array}$ & $\nu_{4} \mathrm{SO}_{4}$ \\
\hline
\end{tabular}

$\mathrm{F}=$ muy fuerte: $\mathrm{f}=$ fuerte: $\mathrm{m}=$ media: $\mathrm{d}=$ debil: $\mathrm{h}-\mathrm{hombro} ; \mathrm{a}=-$ ancha. Las frecuencias $\mathrm{se}$ dan $\mathrm{en} \mathrm{cm}{ }^{1}$. Las bandas mas adecuadas para el análisis se señalan $\mathrm{con} *$.

MATERIALI:S DE CONSTRUCCION N. ${ }^{\circ} 192-1983$ 
$\mathrm{CaSO}_{4}$ (Anhidrita soluble)

\begin{tabular}{|c|c|c|c|}
\hline Frecuencia (cm-1) & Asig. & Frecuencia (cm-1) & Asig. \\
\hline$\left(^{*}\right) 3610$ (débil) & $\nu_{3} \mathrm{H}_{2} \mathrm{O}$ & 1008 (débil) & $\nu_{1} \mathrm{SO}_{4}$ \\
$\left(^{*} 3550\right.$ (débil) & $\nu_{1} \mathrm{H}_{2} \mathrm{O}$ & 665 (media) & \\
$\left(^{*} 3420\right.$ (débil) & & 660 ("hombro") & \\
1156 (fuertte) & & 633 ("hombro") & $\nu_{4} \mathrm{SO}_{4}$ \\
1127 (media) & $\nu_{3} \mathrm{SO}_{4}$ & 625 ("hombro") & \\
1097 (débil) & & 594 (media) & \\
\hline
\end{tabular}

Las bandas señaladas con $\left(^{*}\right)$ se deben a $\mathrm{H}_{2} \mathrm{O}$ y por consiguiente no son caracteristicas de la anhidrita soluble.
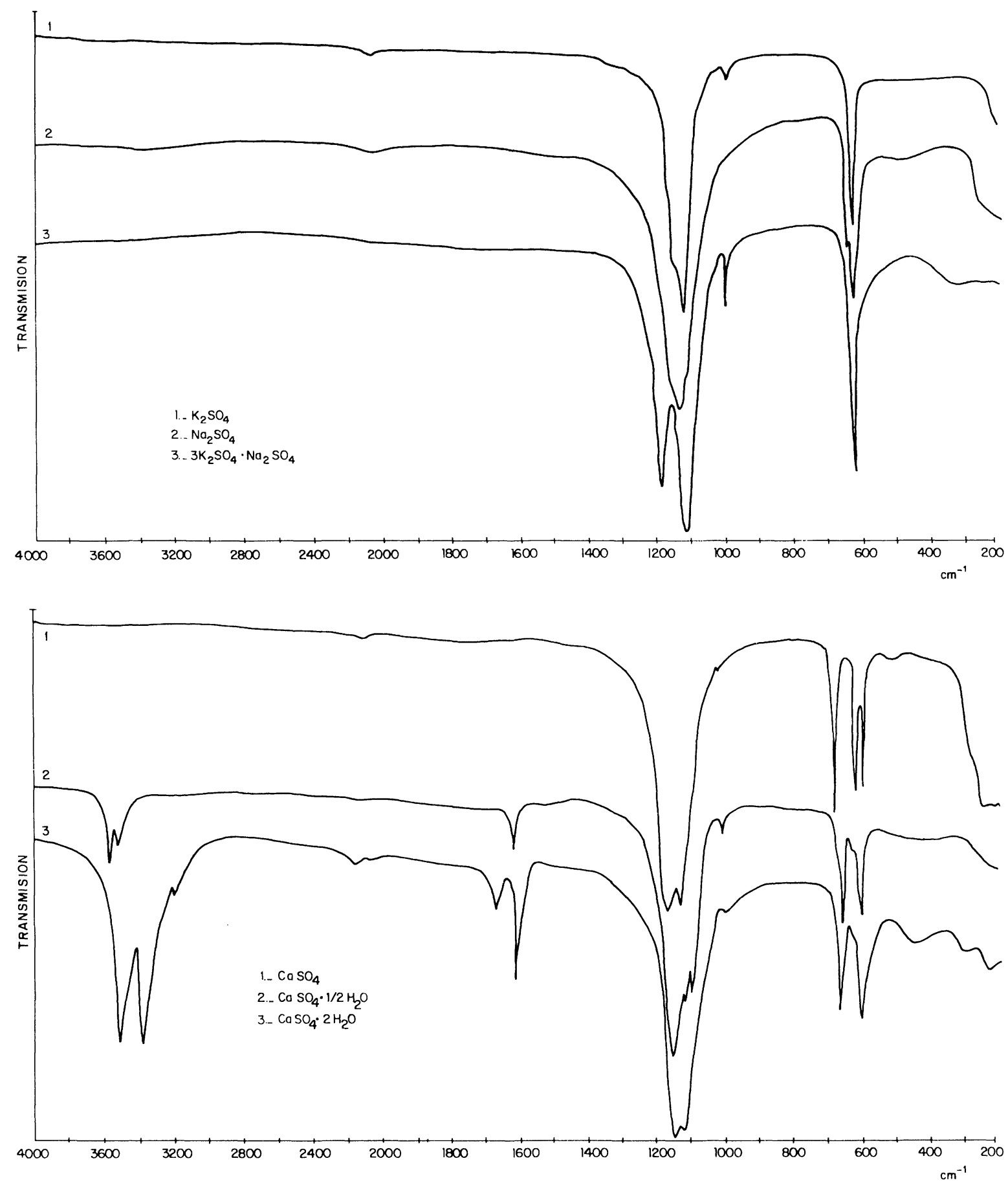

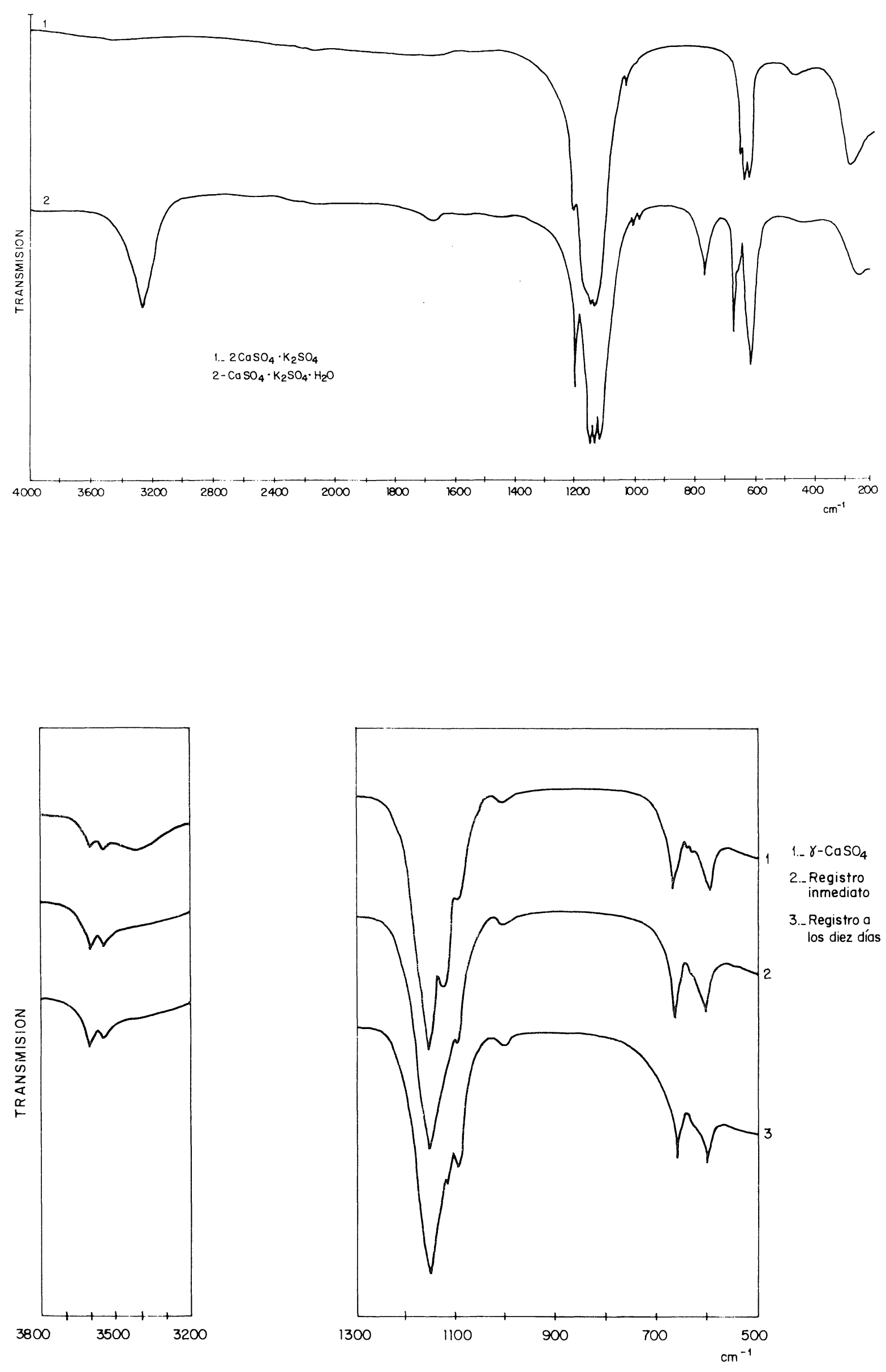

Materiales de Construccion N ${ }^{\circ} 192-1983$ 


\section{B I B L I O G R A F I A}

(1) TAYLOR, W. C.: J. of Research of the N.B.S. vol. (29), 437, (1942).

(2) GOLDMANN, W.; KREFT, W. y SCHUTTE, R.: World Cement Technology 12 (9), 424 (1981).

(3) NEWKIRT, T. F.: III Simposio Internacional de la Quimica del Cemento, LONDRES, 151-167, (1952).

(4) POLLIT, H. W. y BROWN, A. W.: V Simposio Internacional de la quimica de los cementos, TOKYO, vol. I, 322 (1968).

(5) MANDER, J. E. y SKALNY. J. P.: Am. Ceram. Soc. Bull. vol. 56, (11) 987 (1977).

(6) GUTT, W. y SMITH, M. A.: Cement Technology, 143, sep. (1971).

(7) AMAFUJI, M. TSUMAGARI, A.: V Simposio Internacional de la Quimica del Cemento, TOKYO. vol. 1, 136 (1968).

(8) OSBAECK, B.: Cemento y Hormigón, 52, 647 (1981).

(9) MEADE, R. K.: Portland Cement. (1926).

(10) MARGARIT, A. y PUYG, M.: Monografia n. ${ }^{\circ} 220$ del IETCC (1962).

(11) RIDDELL, C. W.: Rock Products, 68, mayo (1950).

(12) TAKEMOTO, K.; ITO, I. y HIRAYAMA, K.: Rock Products, 62, 140, (1959).

(13) BACHIORRINI, A.: Il Cemento 1, 23 (1977).

(14) GILliLAND, J. L.: J. Am. Conc. Inst. 809, junio (1951).

(15) BENSTED, J.: Il Cemento 3, 139 (1975).

(16) POWEL, D. A.: Nature 182, 792 (1958).

(17) MORRIS, R. J.: Nature 198, 1298 (1963).

(18) HAMAD, S. D.: Trans. J. Br. Cera. Soc. 80, 56, (1981).

(19) VAZQUEZ, T. y FERNANDEZ-PEÑA, O.: Cuadernos de investigación del IETCC n.o 34 (1981).

(20) KURDOWSKI, W. y WELISZEK, Z.: VII Congreso Internacional de la Quimica del Cemento, PARIS, vol. 2, 288 (1980).

(21) NIELSEN, H. C.: Rock Products. 77, 2, 72 (1974).

(22) SPRUNG, S.: Zement-Kalk-Gips, 6. 305 (1978)

(23) FOREST, T.: Rev. Mat. Constr. 557, 35 (1962).

(24) MATOUSCHEK, V. F.: Zement-Kalk-Gips, 8, 395 (1972).

(25) RICHARTZ, V. M.: Zement-Kalk-Gips, 2, 67 (1973).

(26) G. PAREDES, P.; CAllejA, J.; VAZQueZ, T. y CEBRIAN, J. L.: Materiales de Construcción 136, 17 (1969).

(27) BENSTED, J.: Il Cemento 3, 117 (1979).

(28) RUIZ DE GAUNA: Materiales de Construcción 152, 5 (1974).

(29) ADAMS, L. D.: Amer. Ceram. Soc. Bụll. 52, 355 (1973).

(30) MANDER, J. E.; ADAMS, L. D. y LARKIN, E. E.: Cem. Conc. Res. 4, 533 (1974).

(31) YAMAGUCHI, G. y TAKAGI, S.: V Simp. Int. de la Quimica del Cemento, TOKYO, vol. I, 181 (1968).

(32) TRIVIÑO, F.: Tésis Doctoral, Madrid (1973).

(33) SKALNY, J. y MAYCOCK, J. N.: J. Am. Ceram. Soc. 57, 253 (1974).

(34) NIEL: Tesis Doctoral, Aachen (1962).

(35) VAZQUEZ, T.: Tesis Doctoral, Madrid (1975).

(36) VAZQUEZ, T.: Materiales de Construcción n.॰ 135 (1969).

(37) BENSTED, T. y VARMA, S. P.: World Cement Tech. 8, 16 (1977).

(38) BACHIORRINI, A. y MURAT. M.: Il Cemento 3, 151 (1976).

(39) Pliego de prescripciones téenicas generales para la recepción de cementos RC-75 (M.O.P. 1975). 
(40) JUGOVIC, Z. T. y GILlAM. J. L.: J. Mater. 3. 517 (1968).

(41) TRIVIÑO, F.: CEMCO-76 del IETCC (1976).

(42) TURRIZIANI. R.: I leganti e il calestruzzo. Roma. (1972).

(43) ENTINE, Z. B. KliOUeVA, L. S. y PAPIA.CHUili, I.: 7. ${ }^{\circ}$ Congreso Int. de la quimica del cemento vol. $2,117$. PARIS (1980).

(44) FRIGIONE, G.: Il Cemento, 3. 207 ( 1 978).

(45) G. PAREDES, P.: Cuadernos de investigación del IETCC n.o 14, 16, 17. 18 y 19 (1968-69).

(46) RIO A. y TURRIZIANI, R.: IV Congreso Int. de la Quimica del Cemento. WASHINGTON (1960).

(47) BOGUE, R. H.: La quimica del cemento portland, New York (1947).

(48) COSTA. V. y MASSAZZA, F.: Il Cemento 4. 181 (1975).

(49) HANSEN. W. C.: IV Congreso Int. de la Quimica del Cemento. WASHINGTON. 387 (1960).

(50) SELIGMANN, P. y GREENING. N. R.: idem. 408-410.

(51) MANABE, T.: idem. 404-408.

(52) SWAIZA. M. A.: idem 416.417

(53) KALOUSEK. G. L.; JUGOVIC. Z. T. y GillaM. J. L.: Cer. Bull. 46. 3. 270 (1967).

(54) SHIN. G. Y. y GLASSER. F. P.: Cement and Concrete Research, v. 13. pp. 135-140 (1983).

\section{publicaciones del i.e.t.c.c.}

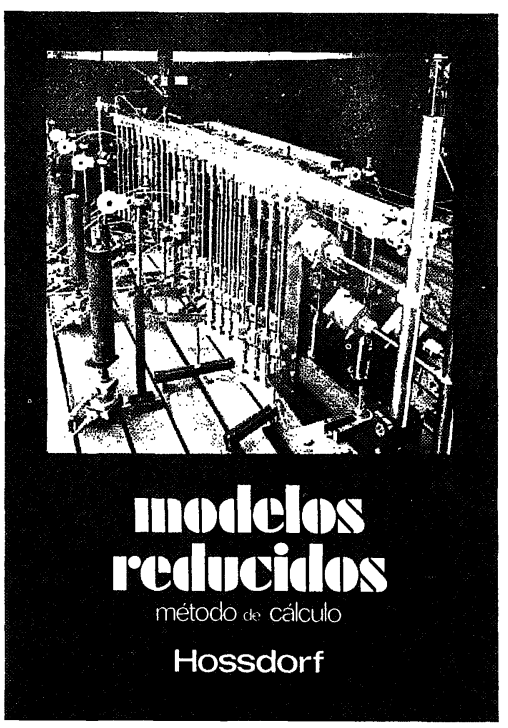

Modelos reducidos. Método de cálculo

H. Hossdorf, Ingeniero.Civil

La técnica de los ensayos en modelos reducidos de estructuras sufre hoy dia una decisiva metamorfosis. hasta hace poco era un medio más bien de artesania, que no siempre era tomado en serio por los académicos teorizantes Dara comprencier el comportaque se acudió las más de las veces, como a un último remedio debido a sus indiscutibles insuficiencias. Sin embargo, en poco tiempo y gracias a su conexión con los ordenadores digitales, se ha trans. formado en un instrumento cientificamente valioso, que no puede quedar a un lado en la práctica diaria del Ingeniero Proyectista.

Un volumen encuadernado en cartoné plastificado con lomo de tela, de $17 \times 24 \mathrm{~cm}$, compuesto de 250 páginas, 158 figuras y fotografias.

Precios: 1.800 ptas.; \$ USA 26.00.

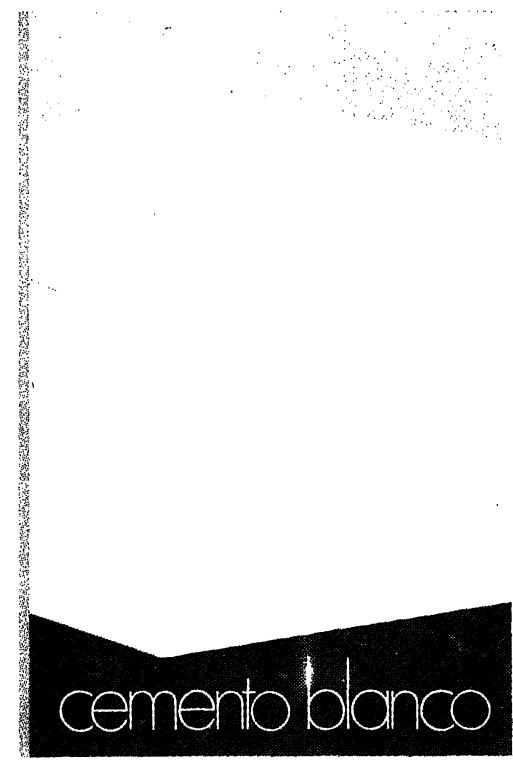

Cemento blanco

Julián Rezola

Ingeniero Quimico Dipl. I. Q. S.

Sabido es que existe una extensa y documentada bibliografia sobre el cemento gris: en cambio, no puede decirse lo mismo acerca del cemento portland sólo a algunas peculiaridades que le distinguen de aquél.

El autor nos ofrece sus profundos conocimientos y su larga experiencia tanto en laboratorio como en fabricación.

La parte descriptiva del libro se complementa con gráficos, diagramas y fotografias de gran utilidad destinados a conseguir la aplicación apropiada de este aglomerante.

Un volumen encuadernado en cartoné policerado, do $17,4 \times 24,3 \mathrm{~cm}$, compuesto de 395 pátginas, numerosas figuras, tablas y ábacos

Precios: España, 1.700 ptas.: extranjero, $\$ 34$.

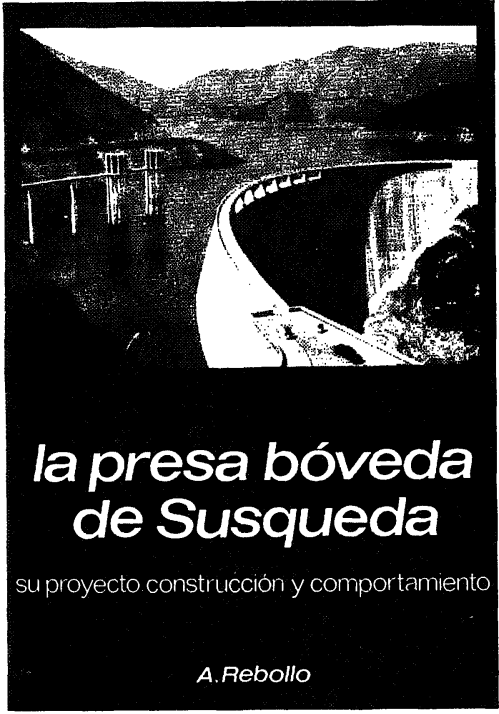

La presa bóveda de Susqueda A. Rebollo, Dr. Ingeniero de Caminos

El esfuerzo del constructor de presas se sitúa, por su pretension de perennidad, a contracorriente de las tendencias de la civilizacion actual, caracte rizada por lo fungible. Pueden evocarse las 10.000 grandes. presas en funcionamiento o en construcción que estan envejeciendo y reclaman los cuidados gerontologicos para mantener y perfeccionar su servicio y garantizar su inalienable pretensión de perennidad. En la medida en que todas nuevas obras, grandes o pequeñas, son portadoras de riesgos ecológicos $y$, a veces, catastróficos, que aumentan con el envejecimiento, la gerontologia de las presas es todo un emplazo. La accion adelantada de Arturo Rebollo en este terreno marca un camino a seguir para todos los que aman su propia obra con la devoción paternal que él ha puesto en Susqueda.

Un volumen encuadernado en cartonè plastificado con lomo de tela, de $18 、 24,5 \mathrm{~cm}$, compuesto de 408 páginas, 330 figuras $y$ fotografias y 39 tablas. Precios: 1.700 ptas.; extranjero, \$ USA 24.00 . 